\title{
A Shifted Power Method for Homogenous Polynomial Optimization over Unit Spheres
}

\author{
Shuquan Wang ${ }^{1}$ \\ ${ }^{1}$ School of Management Science, Qufu Normal University, China. \\ Correspondence: Shuquan Wang, School of Management Science, Qufu Normal University, Rizhao, Shandong, \\ 276800, China.E-mail: msshuquan@163.com
}

Received: March 31, 2015 Accepted: April 21, 2015 Online Published: May 15, 2015

doi:10.5539/jmr.v7n2p175 URL: http://dx.doi.org/10.5539/jmr.v7n2p175

This work was supported by the NSF of China (11171180).

\begin{abstract}
In this paper, we propose a shifted power method for a type of polynomial optimization problem over unit spheres. The global convergence of the proposed method is established and an easily implemented scope of the shifted parameter is provided.
\end{abstract}

Keywords: shifted power method, polynomial optimization problem, convergence

AMS subject classification: 65F99, 65K10, 15A69, 14M15, 90C53

\section{Introduction}

The polynomial optimization problem in which the objective function and constraints are polynomial functions received much attention recently due to their wide applications in such as signal processing (Ghosh 2008, Qi 2003), biomedical engineering (Kofidis 2002, Lasserre 2001), material science (Soare 2008), quantum mechanics (Dahl et al. 2008, Wang et al. 2009), and numerical linear algebra (Hof 2009, Qi 2005), see (Klerk 2008) for a survey on the various classes of polynomial optimization with simplex, hypercube, or sphere constraints. The polynomial optimization problem is a challenging task, as the simplest instances of polynomial optimization, such as maximizing a cubic polynomial over a sphere, is NP-hard (Nesterov 2003). However, researchers have made much contributions in this area from the theoretical side to numerical solution methods (Lathauwer 2000, He 2010, Lasserre 2001, Luo 2010, Qi 2004, 2009, Zhang 2012).

In this paper, we consider the following type of polynomial optimization over unit spheres

$$
\begin{array}{cl}
\min & f\left(\mathbf{x}_{1}, \mathbf{x}_{2}, \cdots, \mathbf{x}_{s}\right) \\
\text { s.t. } & \left\|\mathbf{x}_{i}\right\|^{2}=1, \mathbf{x}_{i} \in \mathbb{R}^{n_{i}} \quad i=1,2, \cdots, s
\end{array}
$$

where $f: \mathbf{R}^{n_{1}} \times \mathbf{R}^{n_{2}} \times \cdots \times \mathbf{R}^{n_{s}} \rightarrow \mathbf{R}$ is a homogenous polynomial function whose each term is also $d_{i}$-order homogenous with respect to $\mathbf{x}_{i}$ for $i=1,2, \cdots, s$.

It is well known that tensor is a useful tool in polynomial optimization as a polynomial, especially a homogenous polynomial, has a very simple expression with the aid of tensor, and furthermore, the optimal condition of a homogenous polynomial optimization with a special structured feasible region can be deeply characterized (Qi 2005, Qi 2009).

A tensor is a multidimensional array of data whose elements are referred by using multiple indices, i.e.,

$$
\mathcal{A}=\left(\mathcal{A}_{i_{1} i_{2} \cdots i_{d}}\right)_{n_{1} \times n_{2} \times \cdots \times n_{d}},
$$

where $d$, the number of indices, is called the order of the tensor, and the $d$-tuple $\left(n_{1}, n_{2}, \cdots, n_{d}\right)$ is called the dimension of the tensor. Tensor $\mathcal{A} \in \mathbf{R}^{n_{1} \times n_{2} \times \cdots \times n_{d}}$ is called super-symmetric if $n_{1}=n_{2}=\cdots=n_{d}$ and $\mathcal{A}_{i_{1} i_{2} \cdots i_{d}}$ is invariant under any permutation of indices $\left(i_{1} i_{2} \cdots i_{d}\right)$, i.e., $\mathcal{A}_{i_{1} i_{2} \cdots i_{d}}=\mathcal{A}_{\pi\left(i_{1} i_{2} \cdots i_{d}\right)}$, where $\pi\left(i_{1} i_{2} \cdots i_{d}\right)$ is a permutation 
of indices $\left(i_{1} i_{2} \cdots i_{d}\right)$. Furthermore, a tensor $\mathcal{A} \in \mathbf{R}^{n_{1} \times n_{2} \times \cdots \times n_{d}}$ is called partially symmetric if its indices can be partitioned into $s$ index blocks and there exist positive integers $d_{1}, d_{2}, \cdots, d_{s}$ such that $d_{1}+d_{2}+\cdots+d_{s}=d$, $n_{1}=n_{2}=\cdots=n_{d_{1}}, n_{d_{1}+1}=\cdots=n_{d_{1}+d_{2}}, \cdots, n_{d_{1}+d_{2}+\cdots+d_{s-1}+1}=\cdots=n_{d}$, and the element $\mathcal{A}_{i_{1} i_{2} \cdots i_{d}}$ is invariant under any permutation of indices within each index block, i.e., $\mathcal{A}_{i_{1} i_{2} \cdots i_{d}}=\mathcal{A}_{\pi\left(i_{1} i_{2} \cdots i_{d_{1}}\right) \pi\left(i_{d_{1}+1} i_{d_{1}+2} \cdots i_{d_{1}+d_{2}}\right) \cdots \pi\left(i_{d_{1}+d_{2}+\cdots+d_{s-1}+1} \cdots i_{d}\right)}$.

By virtue of partially symmetric tensor, problem (1.1) can be written as

$$
\begin{aligned}
\max & \mathcal{A} \mathbf{x}_{1}^{d_{1}} \mathbf{x}_{2}^{d_{2}} \cdots \mathbf{x}_{s}^{d_{s}} \\
\text { s.t. } & \left\|\mathbf{x}_{i}\right\|^{2}=1, \mathbf{x}_{i} \in \mathbb{R}^{n_{i}}, \quad i=1,2, \cdots, s
\end{aligned}
$$

where $\mathcal{A}$ is a partially symmetric tensor w.r.t. index blocks $\left(i_{1} i_{2} \cdots i_{d_{1}}\right),\left(i_{d_{1}+1} i_{d_{1}+2}, \cdots i_{d_{1}+d_{2}}\right), \cdots,\left(i_{d_{1}+d_{2}+\cdots+d_{s-1}+1} \cdots i_{d}\right)$. This problem contains the problem of finding best rank-1 approximation or computing the largest eigenvalue in magnitude of a super-symmetric tensor (Kofidis 2002, Kolda 2011, Zhang 2012, Qi 2009) as special cases. If $s=2$ and $d_{1}=d_{2}=2$, then this problem reduces to the problem considered in (Dah 2008, Wang 2009) arising from the nonlinear elastic materials analysis and entanglement studies in quantum physics. The problem is also a special case of the spherically constrained homogenous polynomial optimization problem considered in (Chen 2012).

Generally, there are three popular numerical solution methods for polynomial optimization. The first one is the SOS approach which is based on the decomposition of a multivariate polynomials into sum of squares (Lasserre 2001, Parrilo 2003), and the second one is the semidefinite programming relaxation technique (Luo 2010) where the concerned problem is approximated by a specially constructed semidefinite programming problem. These two kinds of methods can obtain a global solution of the problem in a sense, however, the computing quantity of these two methods is too large and they are efficient for small scale polynomial optimization problems. The third method is the power method. This method is initiated from the power method for computing the largest eigenvalue in magnitude of a square matrix (Golub 1996), and later was successfully extended to computing the largest singular eigenvalue of a higher-order tensor (Lathauwer 2000) and the largest Z-eigenvalue in magnitude of higher-order super-symmetric tensor (Kofidis 2002, Qi 2005), which in fact are a kind of homogenously polynomial optimization problem with unit sphere constraints (Lathauwer 2000,kofidis 2002). It is also used to compute the largest eigenvalue of a nonnegative tensor. The distinct feature of this method is that it meeds less computing cost at each iteration and its convergence can be guaranteed under convexity assumption. To remove the confine, a novel shifted technique is introduced into the objective function (Kolda 2011,Wang 2009).

In this paper, we extend the shifted power method to a more general type of polynomial optimization problem (1.1) and establish the convergence of the method. The main contribution of the paper is as follows.

(1) We apply the shifted power method to a more general type of polynomial optimization problem defined on the unit spheres and establish its convergence.

(2) We provide an easily implemented scope of the shifted parameters used in the designed iterative method.

The content of this paper is organized as follows. In the Section 2, we will give a short description on the tensor algebra and give some notations. In Section 3, we will give the design power method and establish its convergence.

\section{Preliminaries and Notations}

In this section, we will give a short description on the tensor algebra and some notations used throughout the paper.

For tensors $\mathcal{A}, \mathcal{B} \in \mathbf{R}^{n_{1} \times n_{2} \times \cdots \times n_{d}}$, their inner product is defined by

$$
\langle\mathcal{A}, \mathcal{B}\rangle=\sum_{i_{1}=1}^{n_{1}} \sum_{i_{2}=1}^{n_{2}} \cdots \sum_{i_{d}=1}^{n_{d}} \mathcal{A}_{i_{1} i_{2}, \cdots i_{d}} \mathcal{B}_{i_{1} i_{2}, \cdots i_{d}} .
$$

For vectors $\mathbf{x}_{i} \in \mathbf{R}^{n_{i}}, i=1,2, \cdots, d$, their outer product, denoted by $\mathcal{X}=\mathbf{x}_{1} \circ \mathbf{x}_{2} \circ \cdots \circ \mathbf{x}_{d}$, is a $d$-order $\left(n_{1}, \cdots, n_{d}\right)$ dimensional tensor $\mathcal{T}$ with the $\left(i_{1} i_{2}, \cdots i_{d}\right)$-entry

$$
\mathcal{T}_{i_{1} i_{2} \cdots i_{d}}=\left(\mathbf{x}_{1}\right)_{i_{1}}\left(\mathbf{x}_{2}\right)_{i_{2}} \cdots\left(\mathbf{x}_{d}\right)_{i_{d}},
$$

where $\left(\mathbf{x}_{i}\right)_{i_{r}}$ denotes the $i_{r}$ th entry of vector $\mathbf{x}_{i}$ for $r=1,2, \cdots, n_{i}$.

Based on the inner product of tensors with same order and same size, the inner product of $\mathcal{X}$ with a general tensor $\mathcal{A} \in \mathbf{R}^{n_{1} \times n_{2} \times \cdots \times n_{d}}$ can be expressed as

$$
\langle\mathcal{A}, \mathcal{X}\rangle=\sum_{i_{1}=1}^{n_{1}} \sum_{i_{2}=1}^{n_{2}} \cdots \sum_{i_{d}=1}^{n_{d}} \mathcal{A}_{i_{1} i_{2} \cdots i_{d}}\left(\mathbf{x}_{1}\right)_{i_{1}}\left(\mathbf{x}_{2}\right)_{i_{2}} \cdots\left(\mathbf{x}_{d}\right)_{i_{d}} .
$$


For simplicity, we denote the product by $\mathcal{A} \mathbf{x}_{1} \mathbf{x}_{2} \cdots \mathbf{x}_{d}$. If $\mathcal{A}$ is super-symmetric of dimension $n$ and $\mathbf{x}_{1}=\mathbf{x}_{2}=$ $\cdots=\mathbf{x}_{d}=\mathbf{x} \in \mathbf{R}^{n}$, then the product above is denoted as $\mathcal{A} \mathbf{x}^{d}$. Certainly, $\mathcal{A} \mathbf{x}^{d-1}$ is a $n$-dimensional vector with $i$-th entry

$$
\left(\mathcal{A} \mathbf{x}^{d-1}\right)_{i}=\sum_{i_{2}=1}^{n} \sum_{i_{3}=1}^{n} \cdots \sum_{i_{d}=1}^{n} \mathcal{A}_{i i_{2} i_{3} \cdots i_{d}} \mathbf{x}_{i_{1}} \mathbf{x}_{i_{1}} \cdots \mathbf{x}_{i_{d}},
$$

and $\mathcal{A} \mathbf{x}^{d-2}$ is an $n \times n$ symmetric matrix.

Throughout this paper, we use $\Gamma_{n}$ and $\Sigma_{n}$ to denote the unit ball and sphere in $\mathbf{R}^{n}$, and the subscript $n$ is omitted if no confusion is made.

\section{Algorithm and Convergence}

For problem (1.1), since the linear independence of the constraint qualification is always satisfied, thus for any optimal solution $\left(\mathbf{x}_{1}^{*}, \mathbf{x}_{2}^{*}, \cdots \mathbf{x}_{s}^{*}\right)$, there exists multipliers $\lambda_{i} \in \mathbf{R}, i=1,2, \cdots, s$ such that

$$
\left\{\begin{array}{l}
\mathcal{A}\left(\mathbf{x}_{1}^{*}\right)^{d_{1}-1}\left(\mathbf{x}_{2}^{*}\right)^{d_{2}} \cdots\left(\mathbf{x}_{s}^{*}\right)^{d_{s}}=\frac{\lambda_{1}}{d_{1}} \mathbf{x}_{1}^{*}, \\
\mathcal{A}\left(\mathbf{x}_{1}^{*}\right)^{d_{1}}\left(\mathbf{x}_{2}^{*}\right)^{d_{2}-1} \cdots\left(\mathbf{x}_{s}^{*}\right)^{d_{s}}=\frac{\lambda_{2}}{d_{2}} \mathbf{x}_{2}^{*}, \\
\cdots \cdots \\
\mathcal{A}\left(\mathbf{x}_{1}^{*}\right)^{d_{1}-1}\left(\mathbf{x}_{2}^{*}\right)^{2} \cdots\left(\mathbf{x}_{s}^{*}\right)^{d_{s}-1}=\frac{\lambda_{s}}{d_{s}} \mathbf{x}_{s}^{*} .
\end{array}\right.
$$

This system constitutes the KKT condition of problem (1.2), which corresponds to the stationary point condition of Lagrange function of the problem (Nocedal 1999).

As $\left\|\mathbf{x}_{i}^{*}\right\|^{2}=1$ for $i=1, \cdots, s$, we conclude that

$$
\frac{\lambda_{1}}{d_{1}}=\cdots=\frac{\lambda_{s}}{d_{s}}
$$

Certainly, this is the optimal objective function value at point $\left(\mathbf{x}_{1}^{*}, \mathbf{x}_{2}^{*}, \cdots \mathbf{x}_{s}^{*}\right)$, i.e.,

$$
\frac{\lambda_{i}}{d_{i}}=\mathcal{A} \mathbf{x}_{1}^{* d_{1}} \mathbf{x}_{2}^{* d_{2}} \cdots \mathbf{x}_{s}^{* d_{s}}, \quad i=1,2, \cdots, s .
$$

Based on the shifted power method for computing the largest eigenpairs of a super-symmetric tensor (Kolda 2011, Wang 2009), we have the following shifted power method for solving problem (1.1).

\section{Algorithm 3.1.}

Input: partially symmetric tensor $\mathcal{A} \in \mathbf{R}^{n_{1} \times \cdots \times n_{1} \times n_{2} \times \cdots \times n_{2} \times \cdots \times n_{s} \times \cdots \times n_{s}}$, positive shifted parameters $\alpha_{1}, \alpha_{2}, \cdots, \alpha_{s}$, initial point $\mathbf{x}_{i}^{(0)} \in \mathbf{R}^{n_{i}}, i=1,2, \cdots$, set $k=0$ and take tolerance $\varepsilon \geq 0$.

Output: approximated solution $\mathbf{x}_{1}^{*}, \mathbf{x}_{2}^{*}, \cdots, \mathbf{x}_{s}^{*}$ to problem (1.1)

Iterative Step: FoR $k=0,1,2, \cdots$, Do

$$
\begin{aligned}
& \hat{\mathbf{x}}_{1}^{(k+1)}=\mathcal{A}\left(\mathbf{x}_{1}^{(k)}\right)^{d_{1}-1}\left(\mathbf{x}_{2}^{(k)}\right)^{d_{2}} \cdots\left(\mathbf{x}_{s}^{(k)}\right)^{d_{s}}+\alpha_{1} \mathbf{x}_{1}, \quad \quad \mathbf{x}_{1}^{(k+1)}=\frac{\hat{\mathbf{x}}_{1}^{(k+1)}}{\| \hat{\mathbf{x}}_{k+1)}^{(k+1)}}, \\
& \hat{\mathbf{x}}_{2}^{(k+1)}=\mathcal{A}\left(\mathbf{x}_{1}^{(k+1)}\right)^{d_{1}}\left(\mathbf{x}_{2}^{(k)}\right)^{d_{2}-1} \cdots\left(\mathbf{x}_{s}^{(k)}\right)^{d_{s}}+\alpha_{2} \mathbf{x}_{2}, \quad \mathbf{x}_{2}^{(k+1)}=\frac{\hat{\mathbf{x}}_{2}^{(k+1)}}{\left\|\hat{\mathbf{x}}_{2}^{(k+1)}\right\|} . \\
& \ldots \quad \ldots \quad \ldots \quad \ldots, \quad \ldots \quad \ldots \\
& \hat{\mathbf{x}}_{s}^{(k+1)}=\mathcal{A}\left(\mathbf{x}_{1}^{(k+1)}\right)^{d_{1}}\left(\mathbf{x}_{2}^{(k+1)}\right)^{d_{2}} \cdots\left(\mathbf{x}_{s}^{(k)}\right)^{d_{s}-1}+\alpha_{s} \mathbf{x}_{s}, \quad \mathbf{x}_{s}^{(k+1)}=\frac{\hat{\mathbf{x}}_{s}^{(k+1)}}{\left\|\hat{\mathbf{x}}_{s}^{(k+1)}\right\|} . \\
& \lambda^{(k+1)}=\mathcal{A}\left(\mathbf{x}_{1}^{(k+1)}\right)^{d_{1}}\left(\mathbf{x}_{2}^{(k+1)}\right)^{d_{2}} \cdots\left(\mathbf{x}_{s}^{(k+1)}\right)^{d_{s}} .
\end{aligned}
$$

$\operatorname{IF}\left|\lambda^{(k+1)}-\lambda^{(k)}\right| \leq \varepsilon$, terminate; otherwise, $k=k+1$.

END IF

END FOR 
To see the well-definedness of the algorithm, we need to show that, at each iteration, the gradient of the shifted objective function,

$$
g\left(\mathbf{x}_{1}, \mathbf{x}_{2}, \cdots, \mathbf{x}_{s}\right)=f\left(\mathbf{x}_{1}, \mathbf{x}_{2}, \cdots, \mathbf{x}_{s}\right)+\sum_{i=1}^{s} \alpha_{i} \mathbf{x}_{i}^{\top} \mathbf{x}_{i}=\mathcal{A} \mathbf{x}_{1}^{d_{1}} \mathbf{x}_{2}^{d_{2}} \cdots \mathbf{x}_{s}^{d_{s}}+\sum_{i=1}^{s} \alpha_{i} \mathbf{x}_{i}^{\top} \mathbf{x}_{i}
$$

w.r.t. $\mathbf{x}_{\mathbf{i}}$ on $\Sigma_{n_{1}} \times \cdots \times \Sigma_{n_{s}}$ is bounded away from zero for $i=1,2, \cdots, s$.

Lemma 3.1. There exist $\alpha_{i}>0, i=1,2, \cdots$, s such that the polynomial function

$$
g\left(\mathbf{x}_{1}, \mathbf{x}_{2}, \cdots, \mathbf{x}_{s}\right)=\mathcal{A} \mathbf{x}_{1}^{d_{1}} \mathbf{x}_{2}^{d_{2}} \cdots \mathbf{x}_{s}^{d_{s}}+\sum_{i=1}^{s} \alpha_{i} \mathbf{x}_{i}^{\top} \mathbf{x}_{i}
$$

is strictly convex with respect to $\mathbf{x}_{i}$ on $\Gamma_{n_{1}} \times \cdots \times \Gamma_{n_{s}}$ for $i=1,2, \cdots$, s, and its gradient w.r.t. $\mathbf{x}_{\mathbf{i}}$ on $\Sigma_{n_{1}} \times \cdots \times \Sigma_{n_{s}}$ is bounded away from zero for $i=1,2, \cdots, s$.

Proof. With the notation of the product of tensor, the function $g$ can be expressed as

$$
\begin{aligned}
g\left(\mathbf{x}_{1}, \mathbf{x}_{2}, \cdots, \mathbf{x}_{s}\right) & =\mathcal{A} \mathbf{x}_{1}^{d_{1}} \mathbf{x}_{2}^{d_{2}} \cdots \mathbf{x}_{2}^{d_{s}}+\sum_{i=1}^{s} \alpha_{i} \mathbf{x}_{i}^{\top} \mathbf{x}_{i} \\
& =\mathbf{x}_{i}^{\top}\left(\operatorname{unfold}\left(\mathcal{A} \mathbf{x}_{1}^{d_{1}} \mathbf{x}_{2}^{d_{2}} \cdots \mathbf{x}_{2}^{d_{i}-2} \cdots \mathbf{x}_{2}^{d_{s}}\right) \mathbf{x}_{i}+\alpha I_{n_{i}}\right) \mathbf{x}_{i}+\sum_{i=1, j \neq i}^{s} \alpha_{i} \mathbf{x}_{i}^{\top} \mathbf{x}_{i} .
\end{aligned}
$$

where unfold $\left(\mathcal{A} \mathbf{x}_{1}^{d_{1}} \mathbf{x}_{2}^{d_{2}} \cdots \mathbf{x}_{2}^{d_{i}-2} \cdots \mathbf{x}_{2}^{d_{s}}\right)$ is the unfolded matrix of 2-order tensor in $\mathbf{R}^{n_{i} \times n_{i}}$, and $I_{n_{i}}$ is an identity matrix of dimension $n_{i}$.

To show the strict convexity of function $g\left(\mathbf{x}_{1}, \mathbf{x}_{2}, \cdots, \mathbf{x}_{s}\right)$ w.r.t. $\mathbf{x}_{i}$ on $\Gamma_{n_{1}} \times \cdots \times \Gamma_{n_{s}}$ for $i=1,2, \cdots, s$, it suffices to show that the unfolded matrix of 2-order tensor $\mathcal{A} \mathbf{x}_{1}^{d_{1}} \mathbf{x}_{2}^{d_{2}} \cdots \mathbf{x}_{i}^{d_{i}-2} \cdots \mathbf{x}_{s}^{d_{s}}+\alpha I_{n_{i}}$ is positive definite on $\Gamma_{n_{1}} \times \cdots \times \Gamma_{n_{s}}$. From the partial symmetry of tensor $\mathcal{A}$, this matrix is symmetric, and from the Geršgorin disc theorem (Golub 1996), the spectral radius of matrix $\mathbf{u n f o l d}\left(\mathcal{A} \mathbf{x}_{1}^{d_{1}} \mathbf{x}_{2}^{d_{2}} \cdots \mathbf{x}_{i}^{d_{i}-2} \cdots \mathbf{x}_{s}^{d_{s}}\right)$ is less than or equal to

$$
\sum_{i_{1}, i_{2}, \cdots, i_{d_{1}}=1}^{n_{1}} \sum_{i_{d_{1}+1}, i_{d_{1}+2}, \cdots, i_{d_{1}+d_{2}}=1}^{n_{2}} \cdots \sum_{i_{d_{1}+\cdots+d_{s-1}+1}, \cdots, i_{d}=1}^{n_{d}}\left|\mathcal{A}_{i_{1}, i_{2}, \cdots, i_{d}}\right| .
$$

Denote the sum above as $\alpha$. Then, for any $\alpha_{i}>\alpha$, the matrix

$$
\operatorname{unfold}\left(\mathcal{A} \mathbf{x}_{1}^{d_{1}} \mathbf{x}_{2}^{d_{2}} \cdots \mathbf{x}_{i}^{d_{i}-2} \cdots \mathbf{x}_{s}^{d_{s}}\right)+\alpha_{i} I_{n_{i}}
$$

is positive definite w.r.t. $\mathbf{x}_{1}, \cdots, \mathbf{x}_{s}$ on $\Gamma_{n_{1}} \times \cdots \times \Gamma_{n_{s}}$. From the continuity of the smallest eigenvalue and largest eigenvalue of matrix w.r.t. its elements and the boundedness of $\Gamma_{n_{1}} \times \cdots \times \Gamma_{n_{s}}$, we conclude that the eigenvalue of the above matrix is bounded away from zero on $\Gamma_{n_{1}} \times \cdots \times \Gamma_{n_{s}}$. This means that the matrix is nonsingular w.r.t. $\mathbf{x}_{1}, \cdots, \mathbf{x}_{s}$ on $\Gamma_{n_{1}} \times \cdots \times \Gamma_{n_{s}}$, and thus the gradient of the polynomial function $g\left(\mathbf{x}_{1}, \cdots, \mathbf{x}_{s}\right)$ w.r.t. $\mathbf{x}_{\mathbf{j}}$ on $\Sigma_{n_{1}} \times \cdots \times \Sigma_{n_{s}}$ is bounded away from zero for $j=1,2, \cdots, s$.

Theorem 3.1. For Algorithm 3.1 with $\varepsilon=0$, if $\alpha_{i}$ for $i=1,2, \cdots, k$ satisfies that

$$
g\left(\mathbf{x}_{1}, \mathbf{x}_{2}, \cdots, \mathbf{x}_{s}\right)=\mathcal{A}\left(\mathbf{x}_{1}^{d_{1}} \mathbf{x}_{2}^{d_{2}} \cdots \mathbf{x}_{s}^{d_{s}}+\sum_{i=1}^{s} \alpha_{i} \mathbf{x}_{i}^{\top} \mathbf{x}_{i}\right.
$$

is strictly convex with respect to $\mathbf{x}_{1}, \mathbf{x}_{2}, \cdots, \mathbf{x}_{s}$, then

(1) the generated sequence $\left\{g\left(\mathbf{x}_{1}^{(k)}, \mathbf{x}_{2}^{(k)}, \cdots, \mathbf{x}_{s}^{(k)}\right)\right\}$ is strictly increasing;

(2) if the algorithm terminates after finite steps, then final point is a KKT point of problem (1.1), and if algorithm generate an infinite sequence $\left\{\left(\mathbf{x}_{1}^{(k)}, \mathbf{x}_{2}^{(k)}, \cdots, \mathbf{x}_{s}^{(k)}\right)\right\}$, then

$$
\lim _{k \rightarrow \infty}\left\|\left(\mathbf{x}_{1}^{(k+1)}, \mathbf{x}_{2}^{(k+1)}, \cdots, \mathbf{x}_{s}^{(k+1)}\right)-\left(\mathbf{x}_{1}^{(k)}, \mathbf{x}_{2}^{(k)}, \cdots, \mathbf{x}_{s}^{(k)}\right)\right\|=0
$$

and any accumulation point of $\left\{\left(\mathbf{x}_{1}^{(k)}, \mathbf{x}_{2}^{(k)}, \cdots, \mathbf{x}_{s}^{(k)}\right)\right\}$ is a KKT point of the problem. 
Proof. To prove (1), we first show that for any $k=0,1,2, \cdots$ and $i=1,2, \cdots, s$, it holds that

$$
g\left(\mathbf{x}_{1}^{(k+1)}, \cdots, \mathbf{x}_{i-1}^{(k+1)}, \mathbf{x}_{i}^{(k+1)}, \mathbf{x}_{i+1}^{(k)}, \cdots, \mathbf{x}_{s}^{(k)}\right) \geq g\left(\mathbf{x}_{1}^{(k+1)}, \cdots, \mathbf{x}_{i-1}^{(k+1)}, \mathbf{x}_{i}^{(k)}, \mathbf{x}_{i+1}^{(k)}, \cdots, \mathbf{x}_{s}^{(k)}\right) .
$$

In fact, since the function $g\left(\mathbf{x}_{1}, \mathbf{x}_{2}, \cdots, \mathbf{x}_{s}\right)$ is convex w.r.t. $\mathbf{x}_{i}$ for $i=1,2, \cdots, s$ on $\Gamma_{n_{1} \times \Gamma_{n_{2}} \times \cdots \times \Gamma_{n_{s}}}$, thus for any $i=1,2, \cdots, s$, it holds that

$$
\begin{aligned}
& g\left(\mathbf{x}_{1}^{(k+1)}, \cdots, \mathbf{x}_{i-1}^{(k+1)}, \mathbf{x}_{i}^{(k+1)}, \mathbf{x}_{i+1}^{(k)}, \cdots, \mathbf{x}_{s}^{(k)}\right)-g\left(\mathbf{x}_{1}^{(k+1)}, \cdots, \mathbf{x}_{i-1}^{(k+1)}, \mathbf{x}_{i}^{(k)}, \mathbf{x}_{i+1}^{(k)}, \cdots, \mathbf{x}_{s}^{(k)}\right) \\
\geq & \left\langle\nabla_{\mathbf{x}_{i}} g\left(\left(\mathbf{x}_{1}^{(k+1)}, \cdots, \mathbf{x}_{i-1}^{(k+1)}, \mathbf{x}_{i}^{(k)}, \mathbf{x}_{i+1}^{(k)}, \cdots, \mathbf{x}_{s}^{(k)}\right), \mathbf{x}_{i}^{(k+1)}-\mathbf{x}_{i}^{(k)}\right\rangle .\right.
\end{aligned}
$$

Owning to

$$
\mathbf{x}_{i}^{(k+1)}=\frac{\nabla_{\mathbf{x}_{i}} g\left(\mathbf{x}_{1}^{(k+1)}, \cdots, \mathbf{x}_{i-1}^{(k+1)}, \mathbf{x}_{i}^{(k)}, \cdots, \mathbf{x}_{s}^{(k)}\right)}{\left\|\nabla_{\mathbf{x}_{i}} g\left(\mathbf{x}_{1}^{(k+1)}, \cdots, \mathbf{x}_{i-1}^{(k+1)} \mathbf{x}_{i}^{(k)}, \cdots, \mathbf{x}_{s}^{(k)}\right)\right\|},
$$

one has from the Cauchy-Schwarz inequality that

$$
\left\langle\nabla_{\mathbf{x}_{i}} g\left(\mathbf{x}_{1}^{(k+1)}, \mathbf{x}_{2}^{(k+1)}, \cdots, \mathbf{x}_{i}^{(k+1)}, \mathbf{x}_{i+1}^{(k)} \cdots, \mathbf{x}_{s}^{(k)}\right), \mathbf{x}_{i}^{(k)}-\mathbf{x}_{i}^{(k+1)}\right\rangle \geq 0 .
$$

This means that inequality (3.1) holds.

To show the assertion, we further need to analyze inequality (3.2). For this inequality, as

$$
\nabla_{\mathbf{x}_{i}} g\left(\mathbf{x}_{1}^{(k+1)}, \cdots, \mathbf{x}_{i}^{(k+1)}, \mathbf{x}_{i+1}^{(k)} \cdots, \mathbf{x}_{s}^{(k)}\right) \neq 0, \quad k=0,1,2, \cdots
$$

from Lemma 3.1 and the assumption, by the Cauchy-Schwarz inequality, one has

$$
\left\langle\nabla_{\mathbf{x}_{i}} g\left(\mathbf{x}_{1}^{(k+1)}, \cdots, \mathbf{x}_{i}^{(k+1)}, \mathbf{x}_{i+1}^{(k)}, \cdots, \mathbf{x}_{s}^{(k)}\right), \mathbf{x}_{i}^{(k+1)}-\mathbf{x}_{i}^{(k)}\right\rangle=0
$$

if and only if

$$
\mathbf{x}_{i}^{(k+1)}=\mathbf{x}_{i}^{(k)}=\frac{\nabla_{\mathbf{x}_{i}} g\left(\mathbf{x}_{1}^{(k+1)}, \cdots, \mathbf{x}_{i}^{(k+1)}, \mathbf{x}_{i+1}^{(k)}, \cdots, \mathbf{x}_{s}^{(k)}\right)}{\left\|\nabla_{\mathbf{x}_{i}} g\left(\mathbf{x}_{1}^{(k+1)}, \cdots, \mathbf{x}_{i}^{(k+1)}, \mathbf{x}_{i+1}^{(k)}, \cdots, \mathbf{x}_{s}^{(k)}\right)\right\|} .
$$

From the iterative procedure, (3.4) is also equivalent to

$$
g\left(\mathbf{x}_{1}^{(k+1)}, \cdots, \mathbf{x}_{i-1}^{(k+1)}, \mathbf{x}_{i}^{(k)}, \cdots, \mathbf{x}_{s}^{(k)}\right)=g\left(\mathbf{x}_{1}^{(k+1)}, \cdots, \mathbf{x}_{i-1}^{(k+1)}, \mathbf{x}_{i}^{(k+1)}, \mathbf{x}_{i+1}^{(k)} \cdots, \mathbf{x}_{s}^{(k)}\right) .
$$

Thus, the sequence $\left\{g\left(\mathbf{x}_{1}^{(k+1)}, \mathbf{x}_{2}^{(k+1)}, \cdots, \mathbf{x}_{s}^{(k+1)}\right)\right\}$ is strictly increasing.

For (2), we first consider the case that the algorithm terminates after finite steps, say, stops at Step $k_{0}$. Then from Algorithm 3.1 and discussion for (1),

$$
g\left(\mathbf{x}_{1}^{\left(k_{0}+1\right)}, \mathbf{x}_{2}^{\left(k_{0}+1\right)}, \cdots, \mathbf{x}_{s}^{\left(k_{0}+1\right)}\right)=g\left(\mathbf{x}_{1}^{\left(k_{0}\right)}, \mathbf{x}_{2}^{\left(k_{0}\right)}, \cdots, \mathbf{x}_{s}^{\left(k_{0}\right)}\right)
$$

and

$$
\mathbf{x}_{i}^{\left(k_{0}+1\right)}=\mathbf{x}_{i}^{\left(k_{0}\right)}, \quad i=1,2, \cdots, s .
$$

Combining this with the iterative procedure of Algorithm 3.1 yields that

$$
\nabla_{\mathbf{x}_{i}} g\left(\mathbf{x}_{1}^{\left(k_{0}\right)}, \cdots, \mathbf{x}_{i}^{\left(k_{0}\right)}, \cdots, \mathbf{x}_{s}^{\left(k_{0}\right)}\right)=\left\|\nabla_{\mathbf{x}_{i}} g\left(\mathbf{x}_{1}^{\left(k_{0}\right)}, \cdots, \mathbf{x}_{i}^{\left(k_{0}\right)}, \cdots, \mathbf{x}_{s}^{\left(k_{0}\right)}\right)\right\| \mathbf{x}_{i}^{\left(k_{0}\right)},
$$

i.e.,

$$
\nabla_{\mathbf{x}_{i}} f\left(\mathbf{x}_{1}^{\left(k_{0}\right)}, \cdots, \mathbf{x}_{i}^{\left(k_{0}\right)}, \cdots, \mathbf{x}_{s}^{\left(k_{0}\right)}\right)=\left(\left\|\nabla_{\mathbf{x}_{i}} g\left(\mathbf{x}_{1}^{\left(k_{0}\right)}, \cdots, \mathbf{x}_{i}^{\left(k_{0}\right)}, \cdots, \mathbf{x}_{s}^{\left(k_{0}\right)}\right)\right\|-\alpha_{i}\right) \mathbf{x}_{i}^{\left(k_{0}\right)} .
$$

This means that $\left(\mathbf{x}_{1}^{\left(k_{0}\right)}, \mathbf{x}_{2}^{\left(k_{0}\right)}, \cdots, \mathbf{x}_{s}^{\left(k_{0}\right)}\right)$ is a KKT point of the problem (1.2).

Now, consider the case that the algorithm generates an infinite sequence. In this case, as the sequence $g\left(\mathbf{x}_{1}^{(k)}, \cdots, \mathbf{x}_{s}^{(k)}\right)$ is increasing, we know that it converges to $g^{*}$ as the sequence is defined over unit spheres and thus bounded from above. In particular, one has

$$
\lim _{k \rightarrow \infty}\left(g\left(\mathbf{x}_{1}^{(k+1)}, \cdots, \mathbf{x}_{s}^{(k+1)}\right)-g\left(\mathbf{x}_{1}^{(k)}, \cdots, \mathbf{x}_{s}^{(k)}\right)\right)=0
$$


Taking (3.2) and (3.3) into consideration, we obtain that

$$
\lim _{k \rightarrow \infty}\left\langle\nabla_{\mathbf{x}_{i}} g\left(\mathbf{x}_{1}^{(k+1)}, \cdots, \mathbf{x}_{i}^{(k+1)}, \mathbf{x}_{i+1}^{(k)}, \cdots, \mathbf{x}_{s}^{(k)}\right), \mathbf{x}_{i}^{(k+1)}-\mathbf{x}_{i}^{(k)}\right\rangle=0, \quad i=1,2, \cdots, s .
$$

As

$$
\mathbf{x}_{i}^{(k+1)}=\frac{\nabla_{\mathbf{x}_{i}} g\left(\mathbf{x}_{1}^{(k+1)}, \cdots, \mathbf{x}_{i-1}^{(k+1)}, \mathbf{x}_{i}^{(k)}, \cdots, \mathbf{x}_{s}^{(k)}\right)}{\left\|\nabla_{\mathbf{x}_{i}} g\left(\mathbf{x}_{1}^{(k+1)}, \cdots, \mathbf{x}_{i-1}^{(k+1)}, \mathbf{x}_{i}^{(k)}, \cdots, \mathbf{x}_{s}^{(k)}\right)\right\|}, \quad i=1,2, \cdots, s,
$$

and $\nabla_{\mathbf{x}_{i}} g\left(\mathbf{x}_{1}^{(k+1)}, \cdots, \mathbf{x}_{i-1}^{(k+1)}, \mathbf{x}_{i}^{(k)}, \cdots, \mathbf{x}_{s}^{(k)}\right)$ is bounded away from zero on $\Gamma_{n_{1}} \times \cdots \Gamma_{n_{s}}$, we conclude that

$$
\lim _{k \rightarrow \infty}\left\|\mathbf{x}_{i}^{(k+1)}\right\|^{2}-\left\langle\mathbf{x}_{i}^{(k+1)}, \mathbf{x}_{i}^{(k)}\right\rangle=0, \quad i=1,2, \cdots, s .
$$

Due to that $\left\|\mathbf{x}_{i}^{(k)}\right\|=\left\|\mathbf{x}_{i}^{(k+1)}\right\|=1$, one in turn has

$$
\lim _{k \rightarrow \infty}\left\langle\mathbf{x}_{i}^{(k+1)}, \mathbf{x}_{i}^{(k)}\right\rangle=1, \quad i=1,2, \cdots, s,
$$

and

$$
\lim _{k \rightarrow \infty}\left\|\mathbf{x}_{i}^{(k+1)}-\mathbf{x}_{i}^{(k)}\right\|=0, \quad i=1,2, \cdots, s .
$$

Thus,

$$
\lim _{k \rightarrow \infty}\left\|\left(\mathbf{x}_{1}^{(k+1)}, \mathbf{x}_{2}^{(k+1)}, \cdots, \mathbf{x}_{s}^{(k+1)}\right)-\left(\mathbf{x}_{1}^{(k)}, \mathbf{x}_{2}^{(k)}, \cdots, \mathbf{x}_{s}^{(k)}\right)\right\|=0 .
$$

Since the generated sequence $\left\{\left(\mathbf{x}_{1}^{(k+1)}, \mathbf{x}_{2}^{(k+1)}, \cdots, \mathbf{x}_{s}^{(k+1)}\right)\right\}$ is bounded, by the Bolzano-Weierstrass theorem, the sequence must has at least one accumulation point and these accumulation points constitute a connected set on $\Gamma_{n_{1}} \times \cdots \times \Gamma_{n_{i}}$. Now, we show that each such accumulation point is a KKT point of problem (1.1).

In fact, suppose $\left\{\left(\hat{\mathbf{x}}_{1}, \hat{\mathbf{x}}_{2}, \cdots, \hat{\mathbf{x}}_{s}\right)\right\}$ is an accumulation point of the generate sequence, then there exists subsequence $\left\{\left(\mathbf{x}_{1}^{\left(k_{j}\right)}, \mathbf{x}_{2}^{\left(k_{j}\right)}, \cdots, \mathbf{x}_{s}^{\left(k_{j}\right)}\right)\right\}$ converging to this point. From (3.6), we know that $\left\{\left(\hat{\mathbf{x}}_{1}, \hat{\mathbf{x}}_{2}, \cdots, \hat{\mathbf{x}}_{s}\right)\right\}$ is also a limit of subsequence $\left\{\left(\mathbf{x}_{1}^{\left(k_{j}+1\right)}, \mathbf{x}_{2}^{\left(k_{j}+1\right)}, \cdots, \mathbf{x}_{s}^{\left(k_{j}+1\right)}\right)\right\}$. Replacing $k$ by $k_{j}$ in (3.5) and letting $j \rightarrow \infty$ yield that $\left(\hat{\mathbf{x}}_{1}, \hat{\mathbf{x}}_{2}, \cdots, \hat{\mathbf{x}}_{s}\right)$ is a KKT point of problem (1.1) as discussed in the case of terminating within finite steps.

\section{Conclusion}

In this paper, we proposed a shifted power method for a type of polynomial optimization problem over unit spheres. Its validity is guaranteed theoretically. However, numerical experiments and numerical comparison are required to show the efficiency of the method and this will be done in our future research.

\section{References}

Chen, B., He, S., Li, Z., \& Zhang, S. (2012). Maximum Block Improvement and Polynomial Optimization, SIAM J. Optim., 22, 87-107. http://dx.doi.org/10.1137/110834524

Dahl, G., Leinaas, J. M., Myrheim, J., \& Ovrum, E. (2007). A tensor product matrix approximation problem in quantum physics. Linear Algebra Appl., 420, 711-725. http://dx.doi.org/10.1016/j.laa.2006.08.026

de Klerk, E. (2008). The complexity of optimizing over a simplex, hypercube or sphere: A short survey. Cent. Eur. J. Oper. Res., 16, 111-125. http://dx.doi.org/10.1007/s10100-007-0052-9

de Lathauwer, L., De Moor, B., \& Vandewalle, J. (2000). On the best rank-1 and rank- $\left(R_{1}, R_{2}, \cdots, R_{N}\right)$ approximation of higher-order tensors. SIAM Journal of Matrix Analysis and Applications, 21, 1324-1342. http://dx.doi.org/10.1137/S0895479898346995

Ghosh, A., Tsigaridas, E., Descoteaux, M., Comon, P., Mourrain, B., \& Deriche, R. (2008). A polynomial based approach to extract the maxima of an antipodally symmetric spherical function and its application to extract fiber directions from the orientation distribution function in diffusion MRI, in Proceedings of the Computational Diffusion MRI Workshop (CDMRI08), New York.

Golub, G. H., \& Van Loan, C. F. (1996). Matrix Computations, 3rd ed., Johns Hopkins University Press, Baltimore, MD.

He, S., Li, Z., \& Zhang, S. (2010). Approximation algorithms for homogeneous polynomial optimization with quadratic constraints. Math. Program., 125, 353-383. http://dx.doi.org/10.1007/s10107-010-0409-z 
Hof, P. M. J., Scherer, C., \& Heuberger, P. S. C. (2009). Model-Based Control: Bridging Rigorous Theory and Advanced Technology, Part 1, Springer, New York, 49-68. http://dx.doi.org/10.1007/978-1-4419-0895-7

Kofidis, E., \& Regalia, P. A. (2002). On the best rank-1 approximation of higher-order supersymmetric tensors. SIAM Journal of Matrix Analysis and Applications, 23, 863-884.

Lasserre, J. B. (2001). Global optimization with polynomials and the problem of moments. SIAM J. Optim., 11, 796-817. http://dx.doi.org/10.1137/S1052623400366802

Ling, C., Zhang, X. Z., \& Qi, L. Q. (2012). Semidefinite relaxation approximation for multivariate bi-quadratic optimization with quadratic constraints. Numer Linear Algebra and Applications, 19, 113-131.

Luo, Z. Q., \& Zhang, S. (2010). A semidefinite relaxation scheme for multivariate quartic polynomial optimization with quadratic constraints. SIAM J. Optim., 20, 1716-1736.

Nesterov, Y. (2003). Random Walk in a Simplex and Quadratic Optimization over Convex Polytopes, CORE Discussion Paper, UCL, Louvain-la-Neuve, Belgium.

Nocedal, J., \& Wright, S. J. (1999). Numerical Optimization, New York, Springer.

Parrilo, P. A. (2003). Semidefinite programming relaxations for semialgebraic problems, Mathematical Programming, Series B, 96, 293-320.

Qi, L., \& Teo, K. L. (2003). Multivariate polynomial minimization and its applications in signal processing. J. Global Optim., 26, 419-433.

Qi, L. Q., Wan, Z., \& Yang, Y. F. (2004). Global minimization of normal quartic polynomials based on global descent directions. SIAM J Optim, 15, 275-302.

Qi, L. Q. (2005). Eigenvalues of a real supersymmetric tensor. J Symbolic Computation, 40, 1302-1324. http://dx.doi.org/10.1016/j.jsc.2005.05.007

Qi, L., Wang, F., \& Wang, Y. (2009). Z-eigenvalue methods for a global polynomial optimization problem. Math Programming, 118, 301-316.

Soare, S., Yoon, J. W., \& Cazacu, O. (2008). On the use of homogeneous polynomials to develop anisotropic yield functions with applications to sheet forming. Int. J. Plasticity, 24, 915-944.

Tamara, G. K., \& Jackson, R. M. (2012). Shifted power method for computing tensor eigenvalues. SIAM J Matrix Analysis and Applications, 32, 1095-1124.

Wang, Y. J., Qi, L. Q., \& Zhang, X. Z. (2009). A practical method for computing the largest M-eigenvalue of a fourth-order partially symmetric tensor. Numerical Linear Algebra with Applications, 16, 589-601. http://dx.doi.org/10.1002/nla.633

Zhang, X., Ling, C., \& Qi, L. Q. (2012). The best rank-1 approximation of a symmetric tensor and related spherical optimization problems. SIAM J. Matrix Analysis and Applications, 33, 806-821. http://dx.doi.org/10.1137/110835335

Zhou, G. L., Caccetta, L., Teo, K. L., \& Wu, S. Y. (2012). Nonnegative polynomial optimization over unit spheres and convex programming relaxations. SIAM Journal on Optimization, 22, 987-1008. http://dx.doi.org/10.1137/110827910

\section{Copyrights}

Copyright for this article is retained by the author(s), with first publication rights granted to the journal.

This is an open-access article distributed under the terms and conditions of the Creative Commons Attribution license (http://creativecommons.org/licenses/by/3.0/). 